\title{
Fusion and the Discovery of Isotopes
}

\author{
M. Thoennessen ${ }^{1, \star}$ \\ ${ }^{1}$ National Superconducting Cyclotron Laboratory, Facility for Rare Isotope Beams, and \\ Department of Physics \& Astronomy, Michigan State University, East Lansing, MI 48824, USA
}

\begin{abstract}
.
Heavy-ion fusion-evaporation has been the most productive method to form and identify new isotopes. Of the presently known over 3200 isotopes, almost $25 \%$ were discovered with heavy-ion induced reactions. Since the development of the first heavy-ion beam at Berkeley in 1950 most of the nuclides on the neutron-deficient side of the line of stability and all isotopes of the superheavy elements were discovered utilizing fusion reactions. In addition, some isotopes were first produced in heavy-ion transfer, charge-exchange, incomplete fusion or deep inelastic reactions. The discovery of isotopes relies on new advances in accelerator and detector technology. The continuous development of pioneering and innovative separation and detection techniques have pushed the limit towards - and in many cases beyond - the proton-dripline. A review of the discovery of neutron-deficient and super-heavy nuclides in heavy-ion induced reactions as well as an outlook for the discovery potential in the future is presented.
\end{abstract}

\section{Introduction}

The discovery of new isotopes is the first and necessary step towards the study and exploration of more and more exotic nuclei. Exploring the nuclear landscape and pushing towards the limits of nuclear existence is important for the understanding of the strong force and the nucleosynthesis processes in the universe. More than 3200 different isotopes have been discovered until now. A recent compilation summarized details of the discovery of all isotopes [1-5] including the year, laboratory and country of the discovery as well as the production mechanism used to produce the isotopes. The compilation revealed that more than $25 \%$ of these isotopes were formed in heavy-ion induced fusion reactions making this method the most productive way to discover new isotopes. Figure 1 shows the accumulated number of isotopes discovered as a function of year for the different production mechanisms. Fusion became the dominant production method in the mid 1980s when it overtook light particle reactions. At the present time the only two mechanisms able to reach new isotopes are fusion evaporation and projectile fragmentation reactions.

The current paper presents an overview of the discovery of neutron-deficient and super-heavy nuclides in heavy-ion induced reactions as well as an outlook for the discovery potential in the future. Previously, summaries focusing on the role of fission [6] and the proton dripline [7] have been presented.

\section{Heavy-Ion Accelerators}

The first time the acceleration of heavy-ions was presented at a conference was at the Seattle meeting of the American Physical Society (APS), June 18-21, 1940. Luis Alvarez from the University of California at Berkeley described the acceleration of carbon ions: "The 37-inch cyclotron chamber was filled with $\mathrm{CH}_{4}$ and a beam of $50 \mathrm{Mev}_{6} \mathrm{C}^{12++++++}$ ions was detected with a linear amplifier. To resolve these ions from alpha-particles, it was necessary to reduce the dee voltage and to adjust the magnetic field to the low side of the alpha-particle peak. Under these conditions, about 500 carbon nuclei entered the ionization chamber per minute" [8].

Subsequent progress at Berkeley was reported by Richard Condit two years later: "It has been found possible to produce ${ }^{12} \mathrm{C}^{6}+$ of energy $85 \mathrm{Mev}$ and ${ }^{16} \mathrm{O}^{8}+$ of energy $113 \mathrm{Mev}$ with the 60" Berkeley cyclotron. Very small beams of these high speed particles were led from the cyclotron through a thin window into a Wilson cloud chamber of conventional design" [9].

Another four years later, York et al. presented further improvements at the APS meeting in Berkeley, July 12-13 1946: "Experiments have been done to produce accelerated stripped light nuclei with the 60 " Berkeley cyclotron for use in nuclear experiments. Two types of sources have been investigated: an arc source such as that normally used in cyclotrons and a spark source similar to that used in spectroscopic investigations of highly ionized atoms. Typical yields from an arc source are $1000 \mathrm{C}^{12,+6} 146-\mathrm{Mev}$ ions per second and 100,000 $\mathrm{C}^{12,+6} 135-\mathrm{Mev}$ ions per second..." [10].

\footnotetext{
^e-mail: thoennessen@nscl.msu.edu
} 


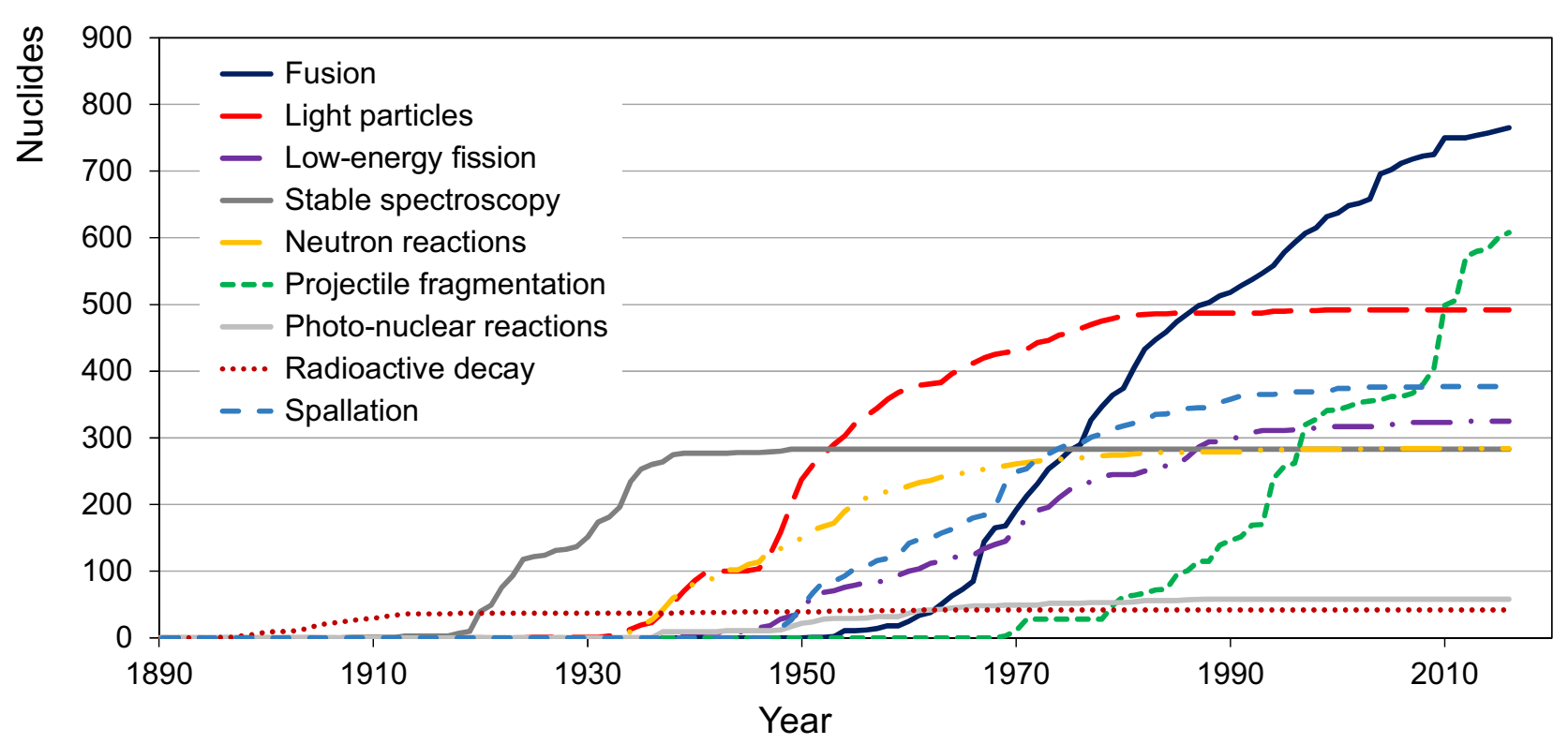

Figure 1. Discovery of nuclides as a function of year. The cumulative sum of the discovered nuclides is shown for different production mechanisms. The line-types and colors are explained in the legend. (Adapted from [4]).

\section{Fusion Evaporation Reactions}

The first successful fusion reactions of two heavy-ions were reported by Miller et al. in the 1950 article published in Physical Review. They bombarded internal aluminum and gold targets and identified ${ }^{34} \mathrm{Cl}$ and ${ }^{205} \mathrm{At}$, respectively: "The nuclear reactions for the production of these two radio-isotopes are presumably $\mathrm{Al}^{27}\left(\mathrm{C}^{12}, \alpha \mathrm{n}\right) \mathrm{Cl}^{34}$, $\mathrm{Au}^{197}\left(\mathrm{C}^{12}, 4 \mathrm{n}\right) \mathrm{At}^{205} "[11]$.

Only a couple of months later Ghiorso discovered the first new isotope with this new technique. They identified ${ }^{246} \mathrm{Cf}$ by irradiating ${ }^{238} \mathrm{U}$ with a beam of $120 \mathrm{MeV}{ }^{12} \mathrm{C}$. The new californium isotope was then formed in the $4 \mathrm{n}$ evaporation channel [12]. It turned out to be the first correct identification of a californium isotope because the earlier published discovery of the element 98 (californium) [13] had misidentified the observed isotope [14].

Soon thereafter it was realized that heavy-ion fusionevaporation reactions were not only an excellent tool to produce new elements but that they also could be used to populate light neutron-deficient nuclei. As an example for a region of current research interest, Figure 2 shows the neutron deficient section of the chart of nuclides in the medium mass region between antimony and bismuth. Proton and $\beta^{+}$-emitters are indicated by the blue and red boxes, respectively. The calculated proton dripline is shown by the thick black line and unknown isotopes which should be in reach to be discovered in the near future are shown as white boxes.

The figure shows that there are several proton-bound even- $Z$ elements that have not been discovered yet. They range from ${ }^{119,120} \mathrm{Ce}$ and ${ }^{123,124} \mathrm{Nd}$, to ${ }^{147,148} \mathrm{Yb}$ and ${ }^{151,152} \mathrm{Hf}$. In addition, for the odd-Z elements there are still unknown isotopes between the last $\beta$-decaying isotope and first proton-emitting isotope. They are ${ }^{118,119} \mathrm{La},{ }^{122,123} \mathrm{Pr}$, ${ }^{132,133} \mathrm{Eu}$, and ${ }^{136,137} \mathrm{~Tb}$. They are difficult to detect because the sensitivity for identifying the $\beta^{+}$decay is not sufficient and the proton decay branch is too weak. In this mass region, promethium is the only odd- $Z$ element for which no proton-emitting isotope has been identified.

The figure also indicates a few isotopes which so far have only been reported in conference proceedings or internal reports (yellow boxes). Souliotis had presented the identification of ${ }^{126} \mathrm{Nd},{ }^{136} \mathrm{Gd},{ }^{138} \mathrm{~Tb}$, and ${ }^{143} \mathrm{Ho},{ }^{150} \mathrm{Yb}$ and ${ }^{153} \mathrm{Hf}$ at the International Conference on Achievements and Perspectives in Nuclear Structure held in Aghia Palaghia, Crete, Greece, July 11-17, 1999 [15], however, the results were subsequently not published in the refereed literature. The observation of ${ }^{143}$ Ho was also reported in an annual report by Seweryniak et al. [16]. In addition, although the discovery of ${ }^{144} \mathrm{Tm}$ was presented at several conferences [17-19] it was never published in a refereed journal.

Over the years fusion-evaporation reactions have become one of the most productive reaction mechanisms to discover new nuclides. In addition to the almost 200 transuranium and superheavy nuclei well over 500 neutron-deficient nuclides were first identified in heavyion fusion evaporation reactions. Since 1951, the 750 isotopes discovered by fusion were observed at 34 different institutions in 16 countries.

Figure 3 shows the chart of nuclides highlighting the isotopes discovered by fusion reactions (red). In addition to a large fraction of neutron-deficient nuclides, all isotopes of the superheavy elements (defined as $\mathrm{Z} \geq 102$ ) were first produced in reactions induced by heavy ions. The figure also shows the isotopes first observed by projectile fragmentation (green). This method dominates the production of exotic neutron-rich nuclides and is the only competing method to produce new nuclides along the proton dripline, especially in the medium mass region. 


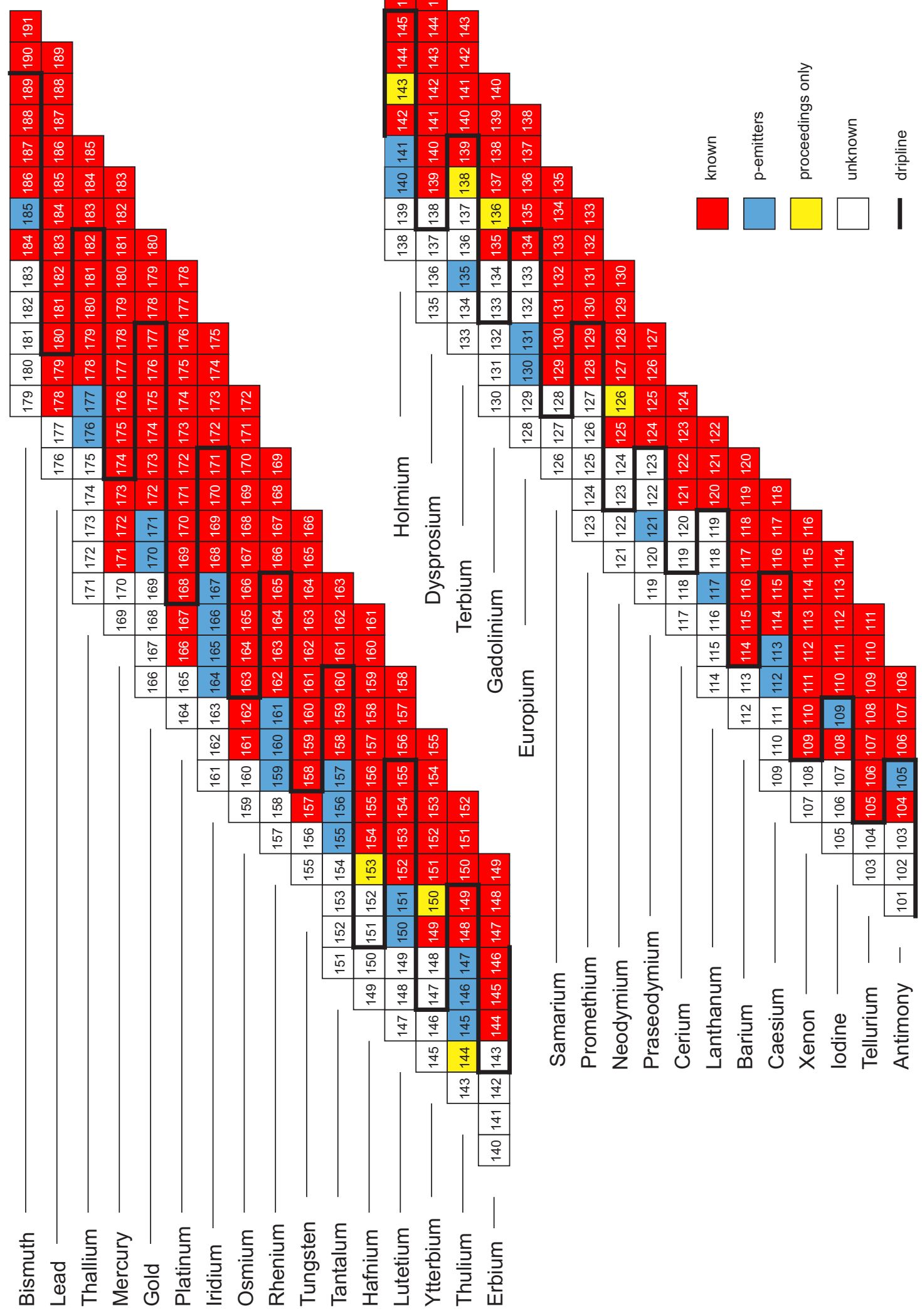

Figure 2. Neutron-deficient chart of the nuclides between antimony and bismuth. The thick black line indicates the proton dripline and the color coding of the isotopes is shown in the legend (Adapted from [3]). 


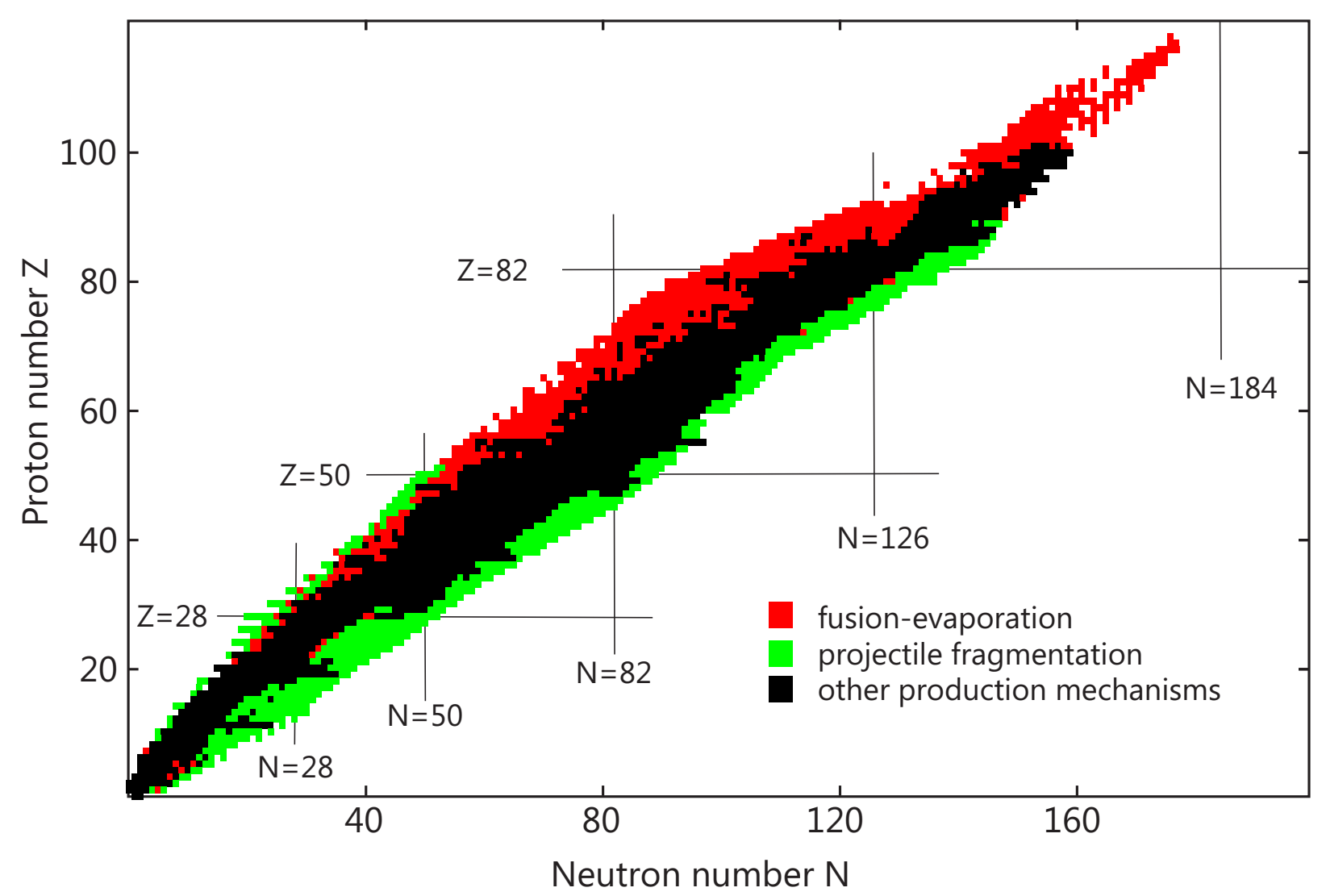

Figure 3. Chart of nuclides. Isotopes discovered by fusion and projectile fragmentation reactions are shown in red and green, respectively. All other known isotopes are shown in black.

Examples of recent discoveries in the heavy mass region are ${ }^{236} \mathrm{Bk}$ and ${ }^{240} \mathrm{Es}$ at Jyväslylä in Finland [20] and ${ }^{230} \mathrm{Am},{ }^{234} \mathrm{Cm}$, and ${ }^{234} \mathrm{Bk}$ at RIKEN in Japan [21]. In addition, ${ }^{178} \mathrm{~Pb}$ was also observed last year at Jyväslylä [22]. Most recently the proton unbound nuclide ${ }^{17} \mathrm{Na}$ was discovered at Michigan State University [23].

\section{Discovery potential}

Although most of the yet to be discovered isotopes are on the neutron-rich side of the valley of stability, there are probably still several hundred unknown isotopes at and beyond the proton dripline that should be able to be observed and identified in the future. Most of them should be reachable with fusion-evaporation reactions. Figure 4 shows the chart of nuclides indicating all compound nuclides that can be populated in heavy-ion fusion reactions with all possible combinations of stable projectiles and stable targets [24]. These compound nuclides decay then by multiple neutron evaporation populating even more neutrondeficient isotopes.

It was recently shown that in addition to complete fusion evaporation reactions, low-energy heavy-ions can also be used to induce multi-nucleon transfer reactions in order to reach regions of new isotopes. Devaraja et al. reported the observation of five new isotopes by bombarding curium targets with $270 \mathrm{MeV}^{48} \mathrm{Ca}$ ions at GSI in Germany [25]. The population of ${ }^{233} \mathrm{Bk}$ required the addition of one proton and the removal of 16 neutrons from ${ }^{248} \mathrm{Cm} .{ }^{229} \mathrm{Am}$ was then observed in the subsequent $\alpha$-decay chain. The third isotope, ${ }^{233} \mathrm{Am}$ was populated by removing one proton and 24 neutrons. Although the subsequent $\alpha$-decay populated the previously unobserved ${ }^{293} \mathrm{~Np}$ no properties of this isotopes were measured. In addition Devaraja et al. claimed the first observation of ${ }^{216} \mathrm{U}$, however, the discovery of this isotope had been submitted for publication only a month earlier by Ma et al. from Lanzhou, China [26].

Multi nucleon transfer reactions had been proposed for the population of neutron-rich isotopes of superheavy elements by Zagrebaev and Greiner [27]. Thus low-energy heavy-ions might also play a role in the extension of the nuclear chart in this region which is essentially not reachable by any other method. Although fusion of radioactive beams has been suggested as an alternative method to populate isotopes in this region, the current proposed intensities for near-term radioactive beam facilities are not sufficient [28].

\section{Summary}

In conclusion, fusion-evaporation reactions have been the most productive method to produce new isotopes. Although since about 2010 most of the newly discovered isotopes per year have been observed by projectile fragmentation, fusion reactions continue to make significant contributions. Further developments and improvements of ac- 


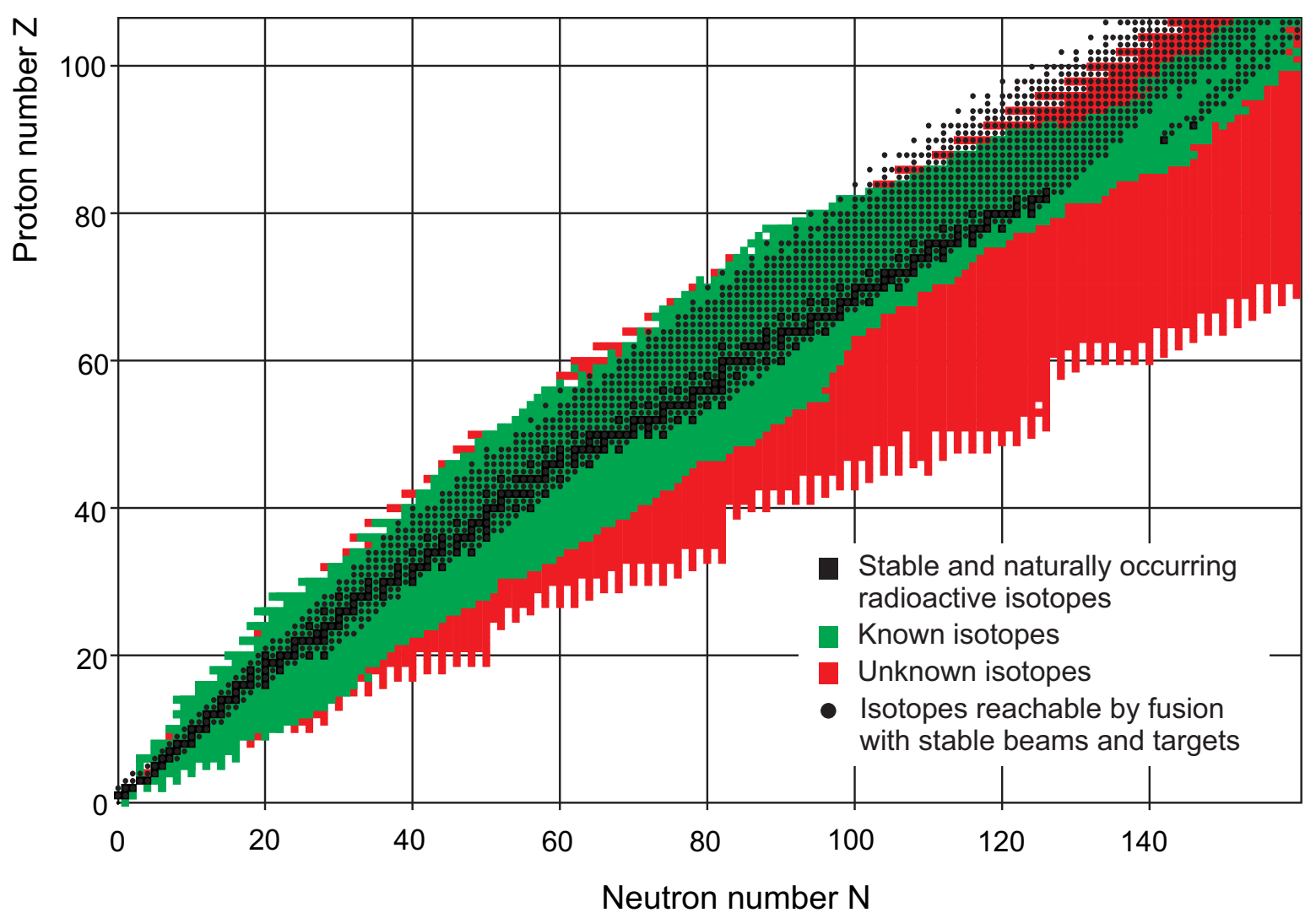

Figure 4. Chart of nuclides: stable (black), presently observed (green) and still unknown (red) nuclides are shown. The small black dots represent compound nuclides that can be populated by fusion reactions with all possible combinations of stable projectiles and stable targets. Dripline nuclides can then be reached by multiple neutron evaporation (Figure adapted from [24]).

celerators and detection and identification techniques are critical for future discoveries to expand the known regions of the nuclear chart. In addition, multi nucleon transfer reactions could also contribute to the observation of new isotopes.

\section{Acknowledgements}

Support of the National Science Foundation under grant No. PHY15-65546 is gratefully acknowledged.

\section{References}

[1] M. Thoennessen, Nucl. Phys. News 22(3), 19 (2012)

[2] M. Thoennessen, Rep. Prog. Phys. 76, 056301 (2013)

[3] M. Thoennessen, Int. J. Mod. Phys. E 25, 1630004 (2016)

[4] M. Thoennessen, The Discovery of Isotopes, A Complete Compilation, Springer International Publishing 2016, doi:10.1007/978-3-319-31763-2

[5] http://www.nscl.msu.edu/ thoennes/isotopes/

[6] M. Thoennessen, Pramana 85, 457 (2015)

[7] M. Thoennessen, Nucl. Phys. Rev. 33, 117 (2016)

[8] L. W. Alvarez, Phys. Rev. 58, 192 (1940)
[9] R. Condit, Phys. Rev. 62, 301 (1942)

[10] H. York et al., Phys. Rev. 70, 446 (1946)

[11] J. F. Miller et al., Phys. Rev. 80, 486 (1950)

[12] A. Ghiorso et al., Phys. Rev. 81, 154 (1951)

[13] S. G. Thompson et al., Phys. Rev. 78, 298 (1950)

[14] A. Chetham-Strode, G. R. Choppin, and B. G. Harvey, Phys. Rev. 102, 747 (1956)

[15] G. A. Souliotis, Physica Scripta T88, 153 (2000)

[16] D. Seweryniak et al., ANL-03/23 (Physics Division Ann. Rept., 2002), p.31 (2003)

[17] R. Grzywacz et al., Eur. Phys. J. A 25, s145 (2005)

[18] K. P. Rykaczewski et al., AIP Conf. Proc. 764, 223 (2005)

[19] C. R. Bingham et al., Nucl. Instrum. Meth. B 241, 185 (2005)

[20] J. Konki et al., Phys. Lett. B 764, 265 (2017)

[21] D. Kaji et al., J. Phys. Soc. Japan 85, 015002 (2016)

[22] H. Badran et al., Phys. Rev. C 94, 054301 (2016)

[23] K. W. Brown et al., Phys. Rev. C 94, 044326 (2017)

[24] M. Thoennessen, Rep. Prog. Phys. 67, 1187 (2004)

[25] H. M. Devaraja et al., Phys. Lett. B 748, 199 (2015)

[26] L. Ma et al., Phys. Rev. C 91, 051302 (2015)

[27] V. Zagrebaev and W. Greiner, Phys. Rev. C 78, 034610 (2008)

[28] W. Loveland, Phys. Rev. C 76, 014612 (2007) 